\title{
Transmission Characteristics of T-ray Multilayer Interference Filters
}

\author{
W. Withayachumnankul, B. M. Fischer, S. P. Mickan, and D. Abbott \\ Centre for Biomedical Engineering and School of Electrical \& Electronic Engineering, \\ The University of Adelaide Adelaide, SA 5005, Australia
}

\begin{abstract}
An optical multilayer interference filter is made from two or more different dielectric materials layered in such a way that it promotes constructive or destructive wave interference for a selected frequency in the direction normal to the layers. Usually, each layer has the thickness of a quarter of wavelength at which the stop-band is required. In this paper, a quarter-wavelength multilayer interference filter is realised for T-ray applications. The dielectric materials used are high-resistivity silicon and free space, both of which have high transparency to T-rays and flat all-pass responses over the frequencies of interest. The designed thickness of both materials is in the order of a hundred microns, and thus allows the novelty of a retrofittable assembled structure. An analysis of the affect of the number of layers on the spectral response is given for the first time. The THz-TDS measurement of the fabricated structure is demonstrated to be in agreement with theory.
\end{abstract}

Keywords: THz-TDS, T-rays, terahertz, interference filter, characteristic matrix

\section{INTRODUCTION}

Ultrafast broadband T-ray systems have opened up the previously inaccessible frequency range lying between millimetre waves and infrared. ${ }^{1}$ Consequently, a range of T-ray components are required to manipulate the propagation of T-rays, inasmuch as optical components are required to control visible light, infrared, or ultraviolet. These components may comprise lenses, mirrors, parabolic mirrors, beam splitters, filters, polarisers, and so on. Most of the T-ray components directly adopt principles from optics. This is possible since the T-ray characteristics are quasi-optical.

One of the common wave-manipulating components is a filter. A number of T-ray filters have been realised to date, owing to the requirements of either conventional FTIR (Fourier Transform Infrared) spectroscopy ${ }^{2}$ or astronomical observations. ${ }^{3}$ These filters can be categorized into two major types, according to the applied optical power, as active or passive filters. An active T-ray filter offers more flexibility in frequency and/or energy tuning, but at the expense of complexity and cost. A passive filter, on the other hand, is less complicated, but also less flexible in terms of its function.

Several approaches to passive terahertz filters are available, for example, reststrahlen bands ${ }^{4}$ particle scattering, ${ }^{5}$ photonic bandgap crystals, ${ }^{6,7}$ perforated metal sheets, ${ }^{8-12}$ and interference in a multilayer structure. A multilayer interference filter* is an attractive option because of its structural simplicity yet optical functionality. By using alternating thin films of T-ray transparent materials, with a proper index arrangement, full control over a particular frequency band is easily attainable.

This work presents a study of the quarter-wavelength multilayer interference filter. The operating frequency range covers the frequency gap of most ultrafast T-ray systems, i.e. between 0.1 and $1.0 \mathrm{THz}{ }^{13}$ Importance is given to the operation in the transmission mode, and the effects of altering the number of filter layers on the transmittance profile. The study of these effects is possible as the submillimetre structure allows a rapid

Email addresses: withawat@eleceng.adelaide.edu.au (W. Withayachumnankul); bfischer@eleceng.adelaide.edu.au (B. M. Fischer); spmickan@eleceng.adelaide.edu.au (S. P. Mickan); dabbott@eleceng.adelaide.edu.au (D. Abbott)

${ }^{*}$ For simplicity in the following context an interference filter refers to a multilayer interference filter and not other types of filters exploiting a similar interference mechanism. The same structure may be found in other optical functions, and/or called by different names such as multilayer periodic structure, dichroic filter, 1D photonic bandgap structure, 1D photonic crystal, Bragg mirror, dielectric mirror, etc.

Photonics: Design, Technology, and Packaging III

edited by Wieslaw Z. Krolikowski, Costas M. Soukoulis, Ping Koy Lam, Timothy J. Davis, Shanhui Fan, Yuri S. Kivshar Proc. of SPIE Vol. 6801, 68011G, (2008) -0277-786X/08/\$18 doi: 10.1117/12.758811

Proc. of SPIE Vol. 6801 68011G-1 
change of the layer arrangement. Characterising of the fabricated filter by THz-TDS delivers a time-domain signal, rarely achievable by other spectroscopic modalities. This evolving time-domain signal reveals significant characteristics of the filter, in addition to the information obtainable from the transmittance profile.

This work is organised as follows: Previous findings on T-ray multilayer interference structures are given in Section 2. Section 3 provides the principle of quarter-wavelength multilayer interference filter. Section 4 shows how the filter response varies as a function of several parameters, including the optical length of each layer, the step between high and low indices of refraction, and the number of periods. Discussion on suitable materials and fabrication methods for the filter is given in Section 5. Experimental results using a fabricated filter are given in Section 6. The work ends with conclusion and potential extensions in Section 7.

\section{REVIEW OF MULTILAYER INTERFERENCE STRUCTURES}

For decades, FTIR spectroscopy has been widely employed in study of materials in the frequency range around the mid-infrared, ${ }^{14}$ usually higher than $1 \mathrm{THz}$. Correspondingly, multilayer interference structures operative in this frequency range, made from a number of different materials and configurations, are widely available. Provided there is no absorption, the structure can be operated either as a filter or mirror dependent on the alignment, as the two functions are complementary. Some multilayer structures used as FTIR mirrors are, for example:

Zinc sulphide/polyethylene ${ }^{15}$ Zinc sulphide $(\mathrm{ZnS})$ layers are deposited onto polyethylene (PE) sheets, the combination of which performs as a narrow band mirror in the 1.5 to $6.0 \mathrm{THz}$ frequency range $\left(n_{\mathrm{ZnS}}=\right.$ $\left.3.0, n_{\mathrm{HDPE}}=1.53, n_{\mathrm{LDPE}}=1.51, \kappa_{\mathrm{PE}}<2 \times 10^{-3}\right)$.

Silicon/air ${ }^{16}$ Multilayer broadband mirrors are constructed from one to six layers of silicon wafers $(n=$ $3.418, l=10 \mu \mathrm{m})$ interleaved by vacuum gaps $(n=1, l=44 \mu \mathrm{m})$. Through FTIR spectroscopy, the mirrors show a nearly constant reflectance spectrum from 1.2 to $3.9 \mathrm{THz}$ and the optimum reflectance at $2.34 \mathrm{THz}$ for the field incident at $40^{\circ}$. The structure was used to build a resonator cavity.

Similar multilayer structures are designed for T-rays, i.e. from 0.1 to $1.0 \mathrm{THz}$, and characterised by THz-TDS. A series of those works are, for example:

Styrolux $^{\mathrm{TM}} / \mathbf{P E}$ and PE/air ${ }^{17}$ An all-plastic mirror is constructed from alternation of $\mathrm{PE}(n=1.74, l=$ $200 \mu \mathrm{m})$ and Styrolux ${ }^{\mathrm{TM}}(n=1.59, l=310 \mu \mathrm{m})$ films. The mirror shows the high reflectivity at the fundamental frequency of $187 \mathrm{GHz}$ with a FWHM of $16 \mathrm{GHz}$. Scattering by the rough film surfaces is responsible for low transmission at high frequencies. To achieve a wider FWHM, it requires two materials with a larger step in the indices of refraction, which are realised by PE films $(n=1.73, l=100 \mu \mathrm{m})$ and air gaps $(n=1, l=200 \mu \mathrm{m})$. The transmittance shows a fundamental stop-band at $386 \mathrm{GHz}$ with a FWHM of $169 \mathrm{GHz}$. The invention is patented for its quarter-wavelength configuration and for operation in the frequency range between $5 \mathrm{GHz}$ and $2 \mathrm{THz} .{ }^{18}$

Polypropylene/silicon ${ }^{19}$ A T-ray omnidirectional mirror is built from five layers of polypropylene $(n=$ $1.53, l=150 \mu \mathrm{m})$ mediated by four layers of high-resistivity silicon $(n=3.418, l=63 \mu \mathrm{m})$. Characterised by THz-TDS in transmission and reflection modes, the mirror can reflect more than $95 \%$ of incident power with frequencies between 319 and $375 \mathrm{GHz}$ at all incidence angles regardless of the polarisation. The structure provides higher reflectivity than does the plastic mirror reported by Turchinovich et al. ${ }^{17}$ By re-sequencing the layers, the structure shows a sharp transmission peak inside a stop-band. ${ }^{20}$

Alumina/alumina-zirconia ${ }^{21}$ A rigid continuous structure is made by tape casting and sintering of the alumina $(n=3.17, l=73 \mu \mathrm{m})$ and alumina-zirconia $(n=4.16, l=51 \mu \mathrm{m})$ into 25 layers. Measured in the transmission mode, this ceramic structure provides a stop-band at 0.3 to $0.38 \mathrm{THz}$ in the normal direction, and a common stop-band at 0.32 to $0.38 \mathrm{THz}$ in all directions. However, due to high loss induced by zirconia, functioning of filter or mirror is limited. 
$\mathbf{P P} / \mathbf{P P}+\mathbf{T i O}_{2}{ }^{22}$ A flexible mirror with a large step of refractive index is made from polymeric compounds. The low-indexed material is polypropylene or PP $(n=1.5, l=269 \mu \mathrm{m})$ and the high-indexed material is $\mathrm{PP}$ with $\mathrm{TiO}_{2}$ additive $(n=3.4, l=111 \mu \mathrm{m})$, both of which are prepared by extrusion. The spectral response of the 5-period mirror shows the centre of reflectivity at $200 \mathrm{GHz}$ with a FWHM of $150 \mathrm{GHz}$ in all directions and polarisations.

These earlier studies focus on finding materials and fabrication techniques suitable for the operation of the multilayer structures in the T-ray frequency range. The fabricated structures are expected to be used as mirrors for short-range or indoor T-ray communication. Thus, particular interest is given to an economic material fabrication, which provides a structure with the highest reflectivity and broadest reflection band at any angle of incidence. Our work, on the other hand, studies the dependency of the structure's characteristics on the number of layers, when the structure is used as a filter in a transmission arrangement. A rapid change of the number of filter's layers is possible owing to the large scale and retrofittability design of the structure. An elegant equation is derived, showing a logarithmic relation between the number of layers and the attenuation within a stop-band. Furthermore, our work supplements full data of the filter's response in terms of time-domain signal and phase response. This serves to provide more insight into the operation of the filter, in addition to characterising the spectral transmittance.

It should be noted that the interference filter can be operated in the reflection mode, to form a the mirror. However, in the reflection mode, a beam splitter is vital if the angle of incidence of the T-rays is normal. Oblique angles eliminate the need of a beam splitter, and thus increase the energy transfer, but the reflectance becomes polarisation-dependent.

\section{QUARTER-WAVELENGTH MULTILAYER INTERFERENCE FILTERS}

A multilayer interference filter is composed of several dielectric layers with different indices of refraction. Cascading these layers to form a periodic structure appropriately, as shown in Figure 1 (a), gives the transmittance profile in the stacking direction similar to that shown in Figure 1 (b). As the name implies, it is the interference mechanism that controls shape and position of the pass-band and stop-band. Particularly, constructive interference is responsible for the pass-band, whereas destructive interference is responsible for the stop-band. To achieve any type of interference, at any desired frequency, the wave dispersion is the only parameter to be adjusted. This is made possible by means of proper material arrangement, given that each material is non-dispersive, i.e. has a constant index of refraction, in the frequencies of interest.

The following subsection briefly discusses the characteristic matrix method, which is required to analyse the response of a multilayer structure. The next subsection explains the required quarter-wavelength optical thickness condition.

\subsection{Characteristic matrix method}

In order to analyse the response of the described periodic structure, the characteristic matrix method ${ }^{23}$ is engaged. In brief, a characteristic matrix for high-indexed material, in case that the wave propagation direction is parallel with the stacking direction, is given by

$$
\mathbf{M}_{H}=\left[\begin{array}{cc}
\cos \left(\frac{\omega}{c} n_{H} l_{H}\right) & j \sin \left(\frac{\omega}{c} n_{H} l_{H}\right) / n_{H} \\
j n_{H} \sin \left(\frac{\omega}{c} n_{H} l_{H}\right) & \cos \left(\frac{\omega}{c} n_{H} l_{H}\right)
\end{array}\right]
$$

where $n_{H}$ and $l_{H}$ are the refractive index and the thickness attributed to a high-indexed material. Likewise, a matrix for the low-indexed material is

$$
\mathbf{M}_{L}=\left[\begin{array}{cc}
\cos \left(\frac{\omega}{c} n_{L} l_{L}\right) & j \sin \left(\frac{\omega}{c} n_{L} l_{L}\right) / n_{L} \\
j n_{L} \sin \left(\frac{\omega}{c} n_{L} l_{L}\right) & \cos \left(\frac{\omega}{c} n_{L} l_{L}\right)
\end{array}\right] .
$$

Here, $n_{L}$ and $l_{L}$ are the refractive index and the thickness attributed to a low-indexed material. When slabs of these materials are layered using $a$ periods, with the high-indexed material terminating both ends, the resulting characteristic matrix is simply obtainable via matrix multiplications in the proper order,

$$
\mathbf{M}_{\text {total }}=\left[\begin{array}{ll}
m_{11} & m_{12} \\
m_{21} & m_{22}
\end{array}\right]=\left(\mathbf{M}_{H} \mathbf{M}_{L}\right)^{a} \mathbf{M}_{H}
$$


The transmission function of the structure in free space is calculated from the total characteristic matrix,

$$
T(\omega)=\frac{2}{m_{11}+m_{12}+m_{21}+m_{22}} .
$$

This transmission function is related to the transmittance via $\mathcal{T}(\omega)=|T(\omega)|^{2}$.

\subsection{Quarter-wavelength optical thickness}

Essentially, due to the destructive interference from reflections, the transmittance of a dielectric slab with the refractive index $n$ and thickness $l$ is minimum at

$$
f_{N}=(2 N+1) \frac{c}{4 n l} ; \quad N=0,1,2, \ldots
$$

This set of minima can be utilised for stop-bands of the filter. However, a single slab cannot cause a wide and deep enough stop-band. Thus, alternate cascading of a number of slabs made from two or more different materials, as illustrated in Figure 1, is required. In order to enhance the stop-bands efficiently, the minima of those slabs must be at the same spectral position. This is made possible through an optical length equalisation:

$$
n_{H} l_{H}=n_{L} l_{L}=\frac{c}{4 f_{0}}=\frac{\lambda_{0}}{4},
$$

where, again, the index of refraction and thickness of the high-indexed material are $n_{H}$ and $l_{H}$, respectively, and those of the low-indexed material are $n_{L}$ and $l_{L}$, respectively. From Equation 6, the optical thickness, $n l$, of a material equals a quarter of the wavelength of the first minimum or the fundamental stop-band. Hence, this formulation leads to the term quarter-wavelength multilayer interference filter.

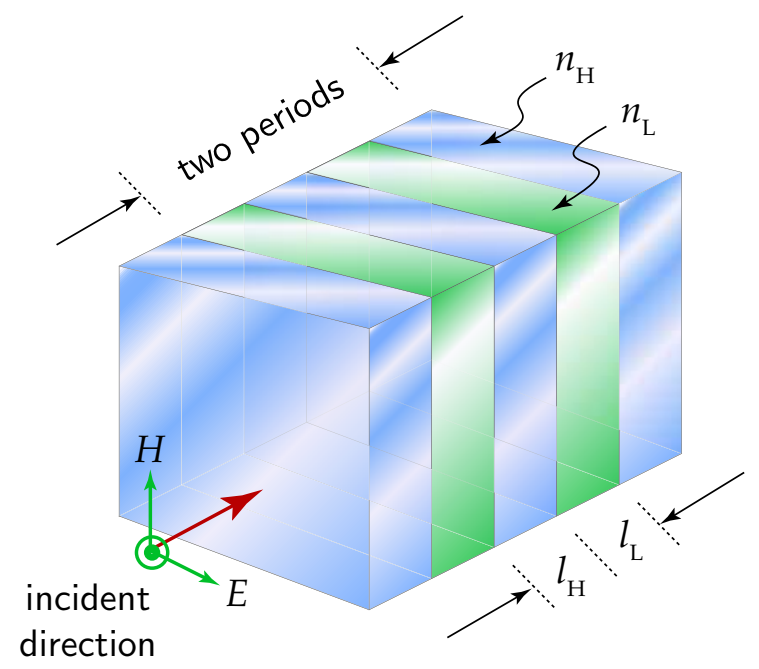

(a) periodic struture, 2.5 periods, $(H L)^{2} H$

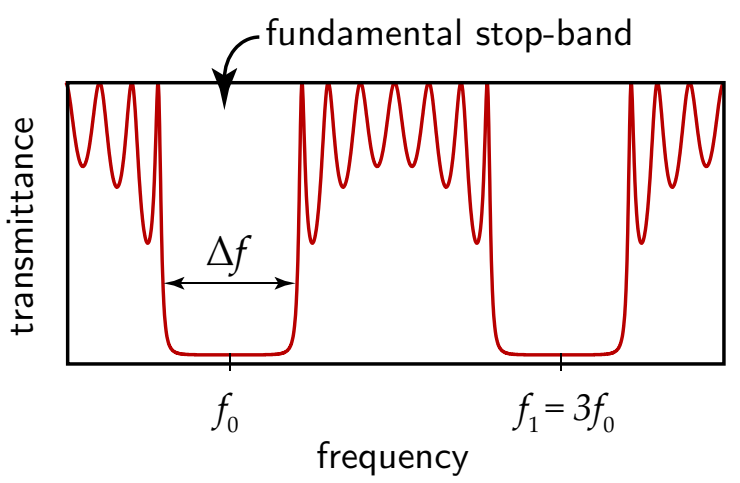

(b) corresponding transmittance

Figure 1. Multilayer interference filter and its transmittance. (a) The structure comprises a number of dielectric periods. Each period is constructed from high- and low-indexed dielectric layers with the indices of refraction of $n_{H}$ and $n_{L}$, and the thicknesses of $l_{H}$ and $l_{L}$, respectively. The periodic structure usually starts and ends with a high-indexed material, and can be designated by $(H L)^{a} H$, where $H$ and $L$ symbolise high- and low-indexed materials, respectively, and $a$ is the number of periods. (b) The transmittance of this periodic structure features spectrally distributed stop-bands. A fundamental stop-band has the central frequency of $f_{0}$ and the effective width of $\Delta f$. 


\section{EFFECTS OF STRUCTURE'S PARAMETERS ON FILTER RESPONSE}

Each parameter of the structural design has effects on the filter spectral response in a unique way. The filter response, including the stop-band position, the width of the stop-band, and the attenuation inside a stop-band, are controlled via these parameters: the optical length of each layer, the step between high and low indices of refraction, and the number of periods. This section analyses and illustrates the relations between the response of a quarter-wavelength interference filter and these factors.

\subsection{Mathematical relations}

From Equation 6, the central frequency of the first stop-band of the filter is given by

$$
f_{0}=\frac{c}{4 n l} .
$$

According to the equation, the optical length $n l$ directly designates the central frequency, $f_{c}$, of stop-bands. Decreasing the optical thickness causes a blue-shift of the central frequency. Changing $n$ and $l$ in reverse proportion has no effect on the frequency $f_{0}$.

Another important property of the filter is the width of a stop-band, $\Delta f$. It is influenced by the step between high and low indices of refraction, and is also scaled by the stop-band position. This relation is given by ${ }^{24}$

$$
\frac{\Delta f}{f_{0}}=\frac{4}{\pi} \arcsin \left(\frac{n_{H}-n_{L}}{n_{H}+n_{L}}\right)
$$

If the difference between the two indices is small, the inverse sine and thus the width of a stop-band approaches zero. If the difference is large, the inverse sine approaches $\pi / 2$, and the stop-band width approaches twice the central frequency. However, in general due to limit of material variation, the width of a stop-band is comparable to the value of the central frequency.

The last filter's property considered here is the attenuation inside a stop-band, in particular the attenuation at $f_{N}$, where $N=0,1,2, \ldots$. Substituting the quarter-wavelength frequency from Equation 7 into Equations 1 and 2 yields, respectively,

$$
\mathbf{M}_{H}=\left[\begin{array}{cc}
0 & j / n_{H} \\
j n_{H} & 0
\end{array}\right] \text {, and } \quad \mathbf{M}_{L}=\left[\begin{array}{cc}
0 & j / n_{L} \\
j n_{L} & 0
\end{array}\right] .
$$

The total matrix is therefore

$$
\mathbf{M}_{\text {total }}=\left(\mathbf{M}_{H} \mathbf{M}_{L}\right)^{a} \mathbf{M}_{H}=(-1)^{a} j\left[\begin{array}{cc}
0 & n_{L}^{a} / n_{H}^{a+1} \\
n_{H}^{a+1} / n_{L}^{a} & 0
\end{array}\right]
$$

where, again, $a$ is the number of periods in the filter. Following Equation 4, the magnitude of the transmission function at $f_{0}$ is

$$
\left|T\left(f_{0}\right)\right|=\frac{2}{n_{L}^{a} / n_{H}^{a+1}+n_{H}^{a+1} / n_{L}^{a}} .
$$

In case that the difference between $n_{H}$ and $n_{L}$ is resonably large, the above equation can be approximated to

$$
\left|T\left(f_{0}\right)\right| \approx \frac{2}{n_{H}}\left(\frac{n_{L}}{n_{H}}\right)^{a} .
$$

Taking a base-10 logarithm of the approximation results in

$$
\log _{10}\left|T\left(f_{0}\right)\right|=a \log _{10}\left(n_{L} / n_{H}\right)-\log _{10}\left(n_{H}\right)+0.3 .
$$

This relation is also applicable to the transmission at $f_{N}$, where $N=1,2,3, \ldots$ and so on. It is obvious that the attenuation in the middle of a stop-band logarithmically depends on the number of periods, $a$. Also note that the attenuation at this frequency is independent of the physical thicknesses of the materials. 

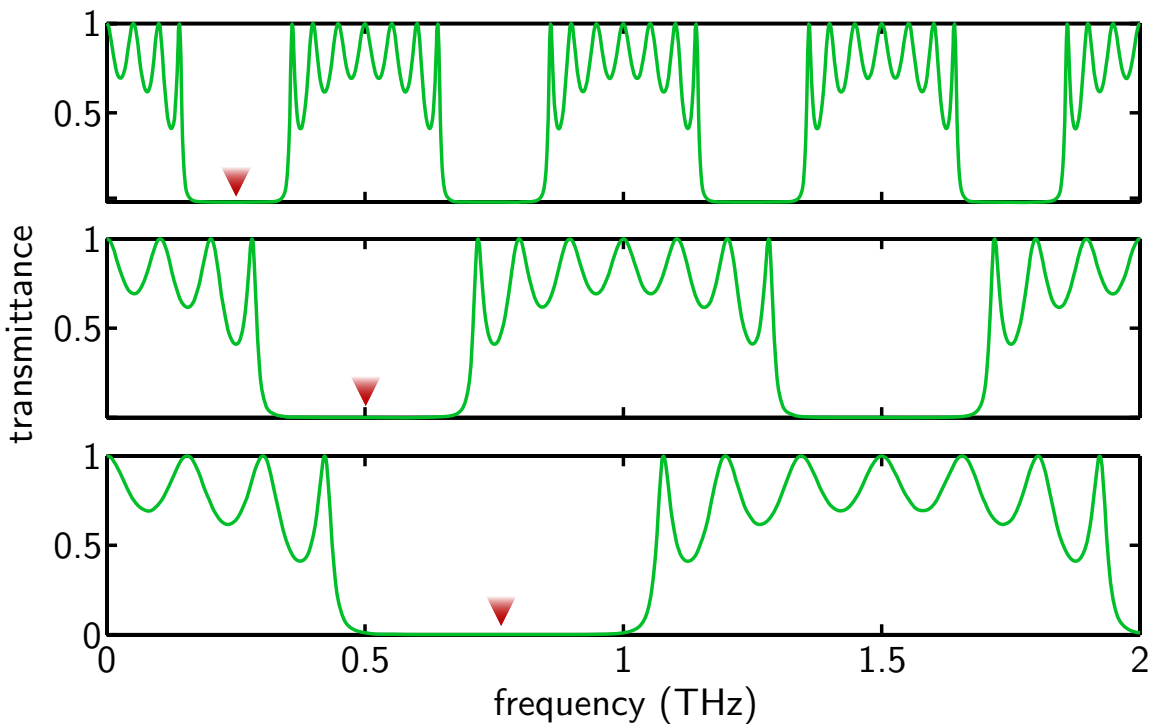

(a)

$n l=300 \mu \mathrm{m}$, or

$f_{0}=0.25 \mathrm{THz}$

(b)

$n l=150 \mu \mathrm{m}$, or

$f_{0}=0.50 \mathrm{THz}$

(c)

$n l=100 \mu \mathrm{m}$, or

$f_{0}=0.75 \mathrm{THz}$

Figure 2. Dependence of filter transmittance on optical thickness. The structure is composed of 3.5 dielectric periods, designated by $(H L)^{3} H$. The high- and low-indexed materials have $n_{H}=3.418$ and $n_{L}=1$, respectively, and their thicknesses are determined from the optical thickness. The arrowheads indicate the position of $f_{0}$.

\subsection{Simulation}

The following simulation confirms the analytical expressions presented in the previous subsection. The simulation helps illustrate the transmittance of the filter affected by changes in the optical thickness, the indices of refraction, and the number of periods. The results are determined on the basis of characteristic matrix analyses, in which the angle of incidence is normal and the transmittance is reported in the range of 0 to $2 \mathrm{THz}$.

Effects of changes in the optical thickness on the central frequency and width of a stop-band are exemplified in Figure 2. Obviously, the figure shows an inverse relation between the optical thickness and the central frequency, as described by Equation 7. When the optical thickness changes from 300, 150, to 100 microns, the central frequency changes from $0.25,0.5$, to $0.75 \mathrm{THz}$, respectively. Also note that the width of a stop-band, $\Delta f$, is scaled in accordance with the central frequency - this relation is explained in Equation 8.

Figure 3 illustrates the transmittance influenced by changes of the materials' indices. Increasing the index difference widens stop-bands, as described in Equation 8. An interesting case is when $n_{H}=3$ and $n_{L}=1$, which allows $\Delta f=f_{0}$. In addition to the stop-band widening, increasing the index difference intensifies the attenuation inside a stop-band, as explained by Equation 13.

Finally, according to Equation 13, the number of periods, a, dictates the attenuation inside a stop-band. Consider Figure 4 where the number of periods evolves from 1.5, 3.5, to 5.5. The attenuation at the middle of a stop-band increases logarithmically, whereas the position and width of a stop-band remain unchanged. In a pass-band, increasing the number of periods results in stronger and more oscillatory transmittance ripples. It is worth noting that the number of layers should be reasonably low to prevent accumulative absorption from bulk material and scattering at material interfaces, which are not considered in the simulation.

\section{MATERIALS AND FABRICATION TECHNIQUES FOR THE FILTER}

This section provides some background discussion on materials and fabrication techniques, needed for realisation of a T-ray interference filter. Fundamental requirements of material properties are similar to those required to realise optic filters, excepting that now the effective frequency range shifts toward the longer wavelength. This results in thicker material layers and completely different fabrication techniques. 


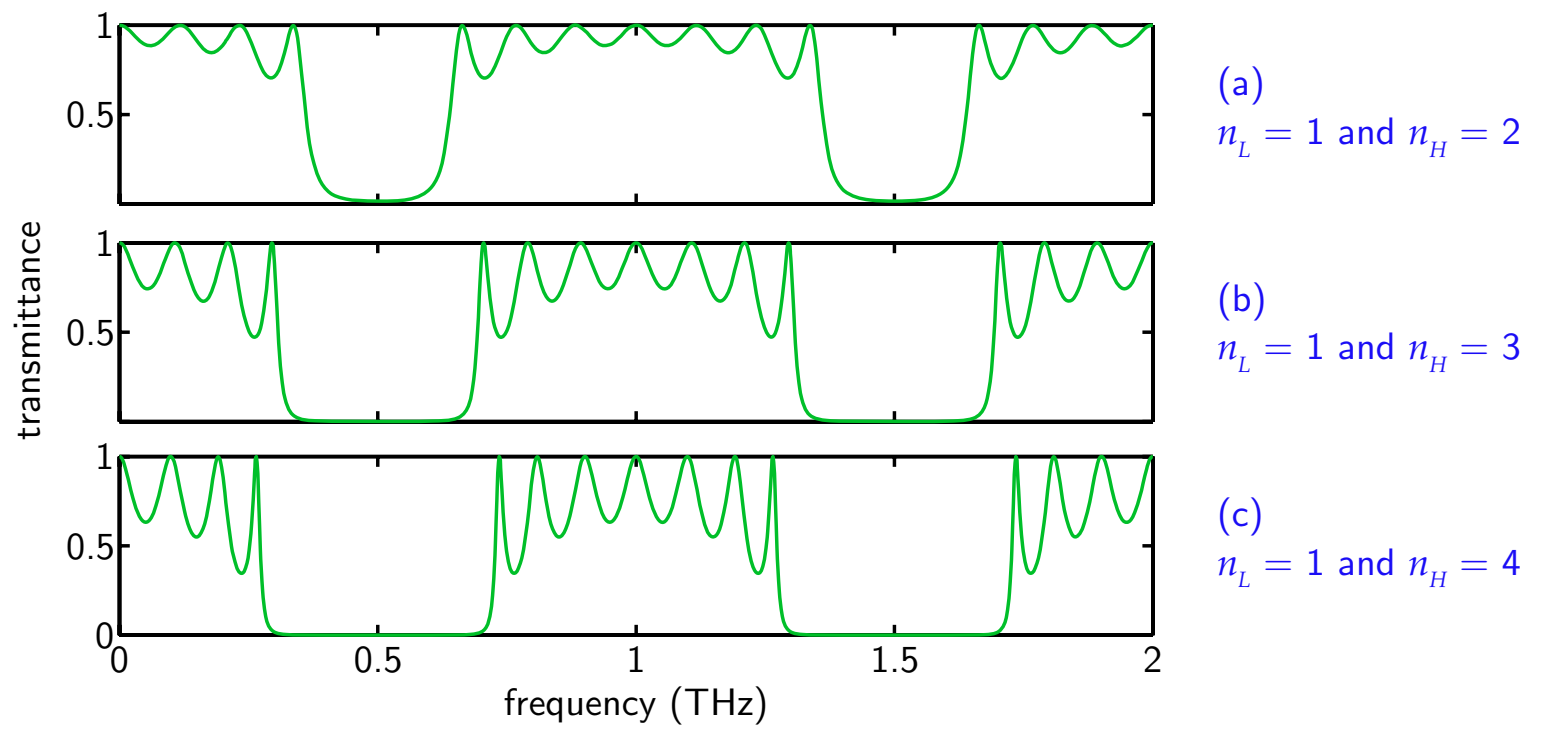

Figure 3. Dependence of filter transmittance on indices of refraction. The structure is composed of 3.5 dielectric periods, designated by $(H L)^{3} H$. The optical length is 150 microns, or the central frequency is $0.5 \mathrm{THz}$, and the physical thicknesses are determined from the optical thickness.

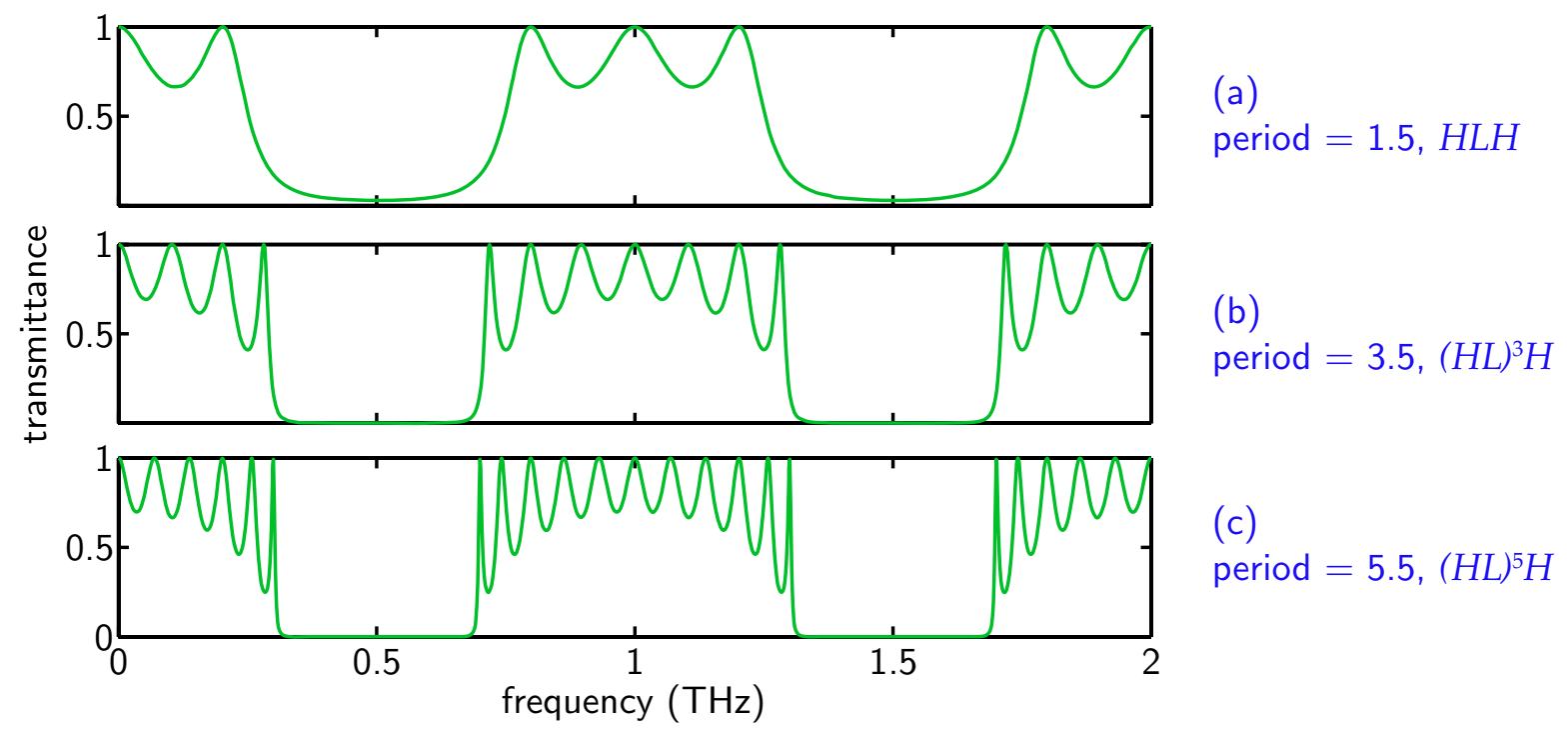

Figure 4. Dependence of filter transmittance on the number of periods. The optical thickness is 150 microns, or the central frequency is $0.5 \mathrm{THz}$. The high- and low-indexed materials have $n_{H}=3.418$ and $n_{L}=1$, respectively, and their physical thicknesses are determined from the optical thickness. 


\subsection{Transparent materials}

As mentioned earlier, a multilayer interference filter exploits the interference mechanism to control the stop-band and the pass-band. High efficiency of the interference mechanism is achievable, provided that each material is non-dispersive and non-absorptive in the T-ray frequency band. The non-dispersiveness of materials facilitates the design and sustains the uniformity of the interference mechanism throughout the frequencies of interest. The non-absorption, including low scattering, of materials avoids energy dissipation, which degrades the transmittance in the pass-band and also heats up the structure undesirably.

In addition to the absorptive and dispersive properties of materials, there are a number of other relevant factors, e.g. mechanical stability, chemical resistivity, thermal conductivity, birefringence, temperature dependency, or optical transparency. Though, the importance of each factor depends on a specific application. At this stage, for ordinary filter tasks, the interest is principally in the absorptive and dispersive properties of the material. In addition, some care is necessary to control mechanical stability and birefringence.

Two dielectric materials, which reasonably satisfy the above requirements and have a large difference in refractive index, are silicon and dry air. The first material, undoped high-resistivity float-zone (FZ) silicon, is known to have a negligible absorption and a constant refractive index of 3.418 at T-ray frequencies. ${ }^{25,26}$ The second material, dry air, has a unity refractive index and zero absorption for T-rays. Both silicon and air have no birefringence, and the optical properties of silicon in the T-ray regime are independent of the crystal orientation.

\subsection{Fabrication techniques}

From the quarter-wavelength condition in Equation 6, it can be seen that the wavelength of T-rays in the submillimetre region requires the dielectric layers to have the same order of thickness. This thickness requirement excludes most of the conventional methods, used for fabrication of optical components, because those methods are optimised for coating in the order of a few microns. Some other potential fabrication methods are suggested for T-ray components, for example:

Material adhesion assembles different dielectric materials by means of either a holder, adhesive, or thermal bond. The applicability of these methods depends on the types of materials in use. Examples of the structures that are assembled by these methods are, for instance, Turchinovich et al. ${ }^{17}$ or Krumbholz et al. ${ }^{19}$

Material deposition employs a chemical process to deposit desired materials onto a substrate. This technique requires complicated deposition equipment. Furthermore, a practical deposit thickness is often in an order of a few microns, which is too thin for use with T-ray wavelengths. A thicker layer is possible at the cost of deposition time and mechanical instability.

Hybrid method utilizes both material adhesion and material deposition in the fabrication. A suitable material is deposited onto a thin film, which is, afterwards, stacked with other deposited films to form a multilayer periodic structure. This method is used by, e.g. Shao and Dobrowolski. ${ }^{15}$

Air-gap method, a special case of material adhesion, eliminates the requirement for a second material of different index of refraction by substitution of air or vacuum, which has an index of refraction of approximately unity. In addition to the constant index, this method has an advantage concerning a low effective absorption, since free space has no absorption at all frequencies or can be considered as an all-pass filter. Another advantage is that the problem of unintended air-gaps, ${ }^{19}$ present between layers, vanishes. Implementations of this method can be found in, for example, Turchinovich et al. ${ }^{17}$ or Schiwon et al. ${ }^{16}$ For the above reasons, the air-gap method is also preferred in this work.

Other fabrication techniques suitable for constructing interference filters are, for example, plastic coextrusion or tape casting and sintering of ceramics. ${ }^{21}$

For visible light and nearby frequencies, according to Equation 6, the thickness of each layer is in the order of submicrons, and thus a rigid substrate is required in order to support the periodic structure. However, this is not true for T-rays for which the interference layer thickness is around a few tens to a few hundreds of microns. Therefore, all of the layers can be stacked to form a free-standing structure, and a substrate is unnecessary for 


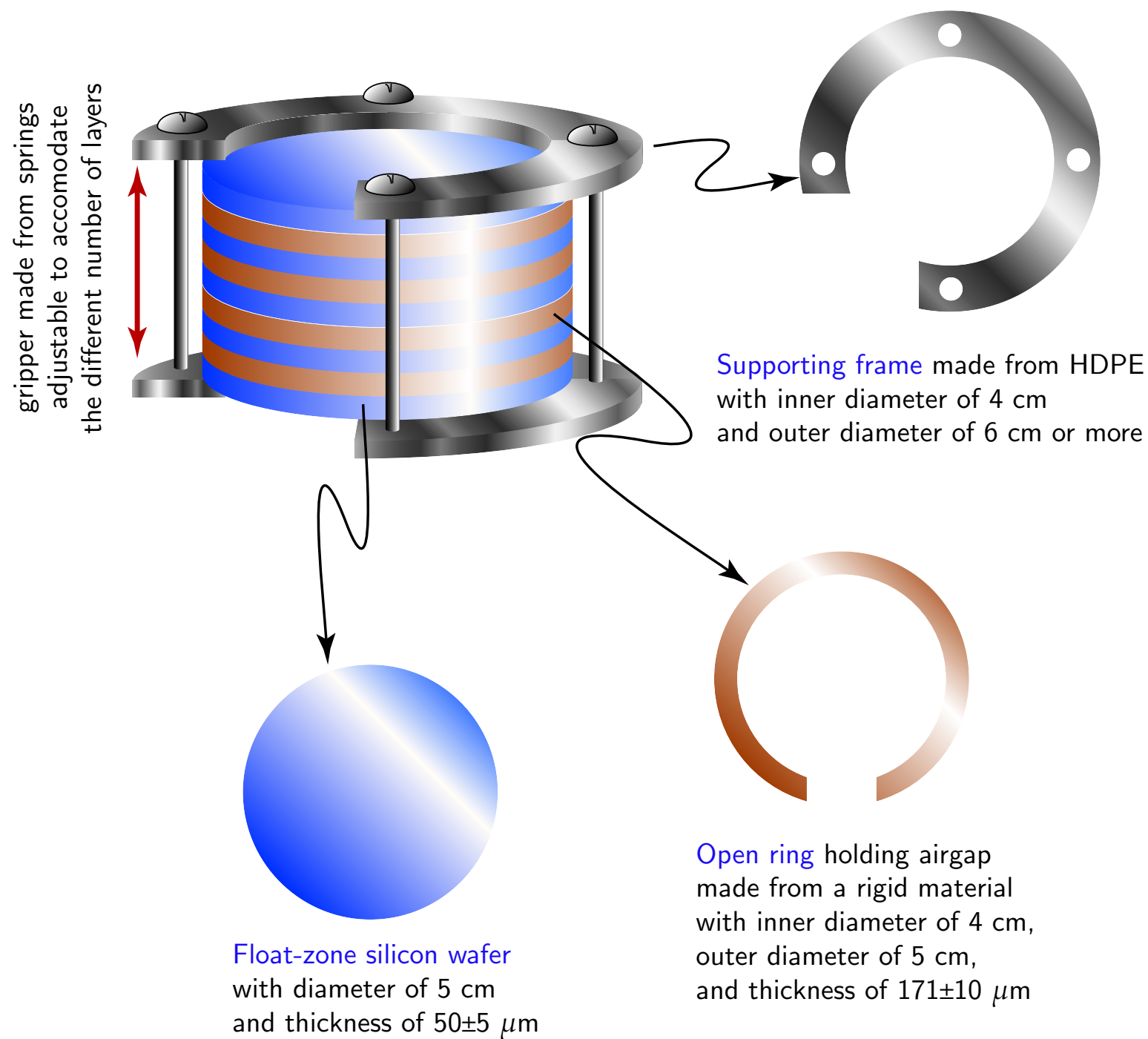

Figure 5. Design of multilayer interference filter. The filter is composed of FZ silicon wafers alternating with air gaps, created by open rings. The materials are held together by a supporting frame made from high-density polyethylene (HDPE), and therefore the layers can be replaced instantaneously. The diameter of the structure is wide enough, so that the T-ray beam can pass through without edge diffraction.

the T-ray interference filter. This is beneficial because (i) the Fabry-Pérot fringes induced by a thick substrate can be easily removed from the spectrum, and (ii) the overall absorption of the structure is reduced. In addition, a submillimetre thickness suggests the retrofittability feature of the assembled structure, which allows a change to the structural arrangement with ease.

\section{EXPERIMENT AND RESULT}

\subsection{Design of the silicon-air filters}

A batch of undoped FZ silicon wafers supplied by Siltronix, each with a thickness of $50 \pm 5 \mu \mathrm{m}$, is utilised. Due to the Fabry-Pérot effect, or particularly the destructive interference, the transmission of a single wafer has a minimum at $f_{0}=c /\left(4 n_{\mathrm{si}} l_{\mathrm{si}}\right)=0.439 \mathrm{THz}$. This frequency is then set as the central frequency of the fundamental stop-band. The thickness of an air gap, $l_{\text {air }}=170.9 \mu \mathrm{m}$, is chosen to comply with the quarter- 


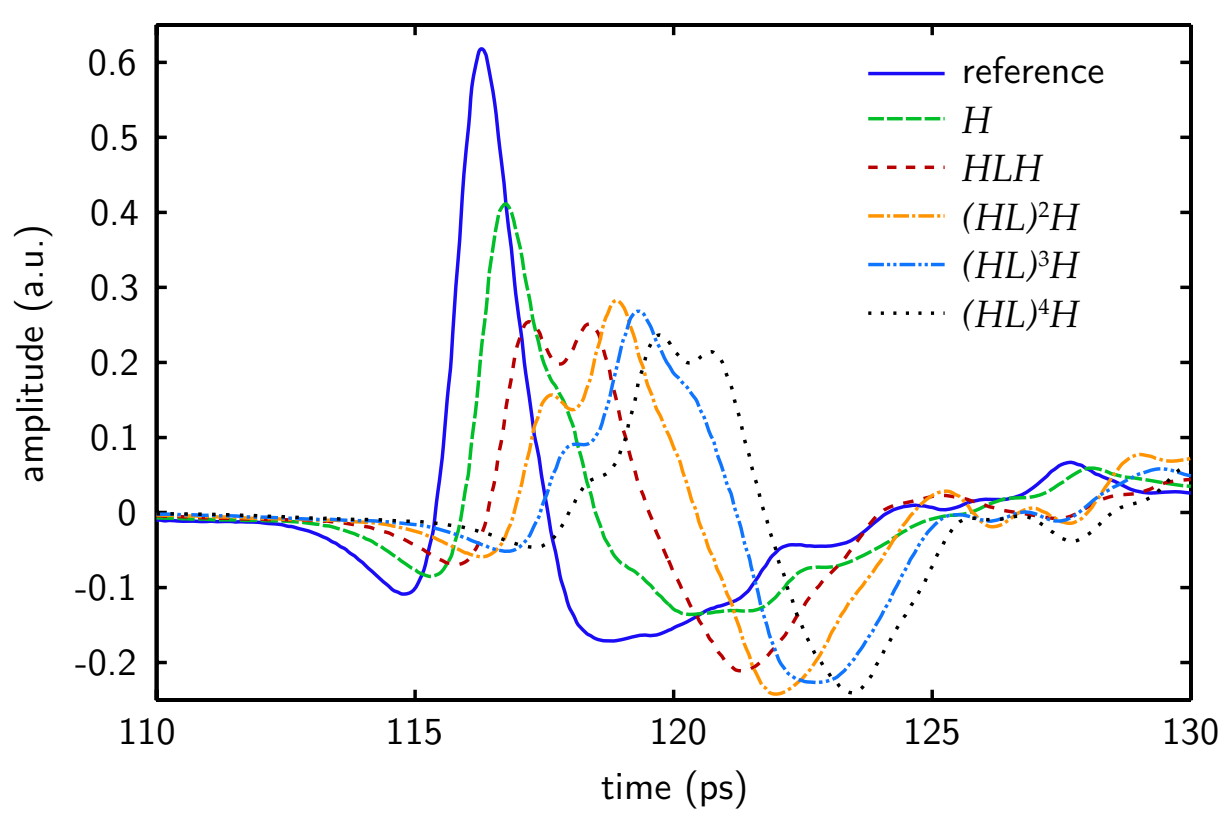

Figure 6. T-ray signals transmitted through the interference filters. Only the signals inside the 20-ps window from the total recording duration of $555.22 \mathrm{ps}$ are shown. The $H$ measurement in fact represents the signal recorded from a single 50-micron silicon wafer. The pulses are delayed, attenuated, and reshaped by the filters.

wavelength condition in Equation 6. Given $n_{\mathrm{si}}=3.418$ and $n_{\text {air }}=1$, the width of a stop-band is estimated at $0.32 \mathrm{THz}$, according to Equation 8.

The material layers, comprising silicon wafers and air-rings, are fitted together by a HDPE frame, which exerts pressure on the structure via the elastic force of springs. This spring-tight design helps reduce uneven forces at different surface positions and for different numbers of layers. Also it facilitates the replacement and retrofitting of material layers. An illustration of the design is given in Figure 5

\subsection{Characteristics of the silicon-air filters}

A series of silicon-air filters with various periods are characterised by transmission THz-TDS, with a spectrometer having a bandwidth from 0.1 to $1.0 \mathrm{THz}$ and the maximum dynamic range of $25 \mathrm{~dB}$. The measurement for each filter is carried out nine times, and then the time-domain pulses are averaged. Also, the reference signal measured with the same setting is collected regularly to compensate amplitude drifting effects of the system. For each recorded signal, the temporal window is $555.22 \mathrm{ps}$ with the sampling interval of 33.9 fs. The Fourier transform of the pulse yields a spectral resolution of $1.8 \mathrm{GHz}$.

Figure 6 shows a set of pulses recorded from the silicon-air interference filters with various numbers of siliconair periods. Determined from the first zero crossing point, each signal is delayed from its neighbour by $0.5 \mathrm{ps}$. The greater delays of the pulses are attributed to the increasing effective indices of refraction of the structures. The pulses for $(H L)^{a} H$ structures exhibit very little difference in the maximum amplitudes, which are approximately $40 \%$ of the reference amplitude. This is because the filters attenuate the amplitude at the specific frequency range while preserving the rest. However, the pulse shapes among the filters are largely distinctive. A possible likely cause is the spectral ripple in the pass-band, which is shown in the transmittance profiles (Figure 7).

The transmittances in Figure 7 are evaluated from the spectra of the reference, $E_{\text {ref }}(\omega)$, and of the filter, $E_{\text {fil }}(\omega)$, using $\mathcal{T}(\omega)=\left|E_{\text {fil }}(\omega) / E_{\text {ref }}(\omega)\right|^{2}$. From the figure, the first stop-band locates between 0.2 and $0.5 \mathrm{THz}$, the centre of which is at approximately $0.36 \mathrm{THz}$, and the FWHM is $\approx 0.3 \mathrm{THz}$. As the period of the filters increases, the attenuation in the middle of the stop-band increases logarithmically, roughly at an order of magnitude per period. This logarithmic relation confirms the theory in Equation 13, and is plotted explicitly in Figure 8. The filters have ripples in the pass-band, i.e. between 0.0 and $0.2 \mathrm{THz}$, and between 0.5 to $0.9 \mathrm{THz}$, which are stronger 


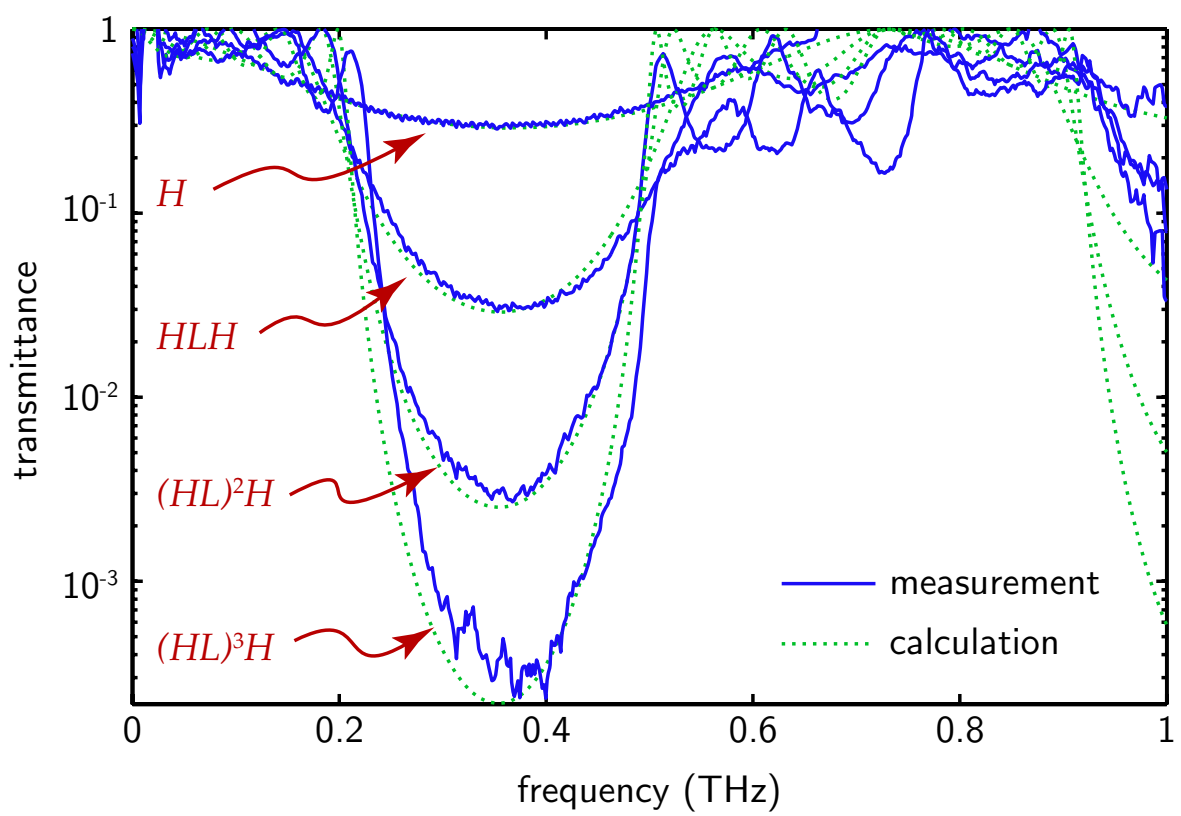

Figure 7. Transmittance of the interference filters. For each filter, the first stop-band resides between 0.2 and $0.5 \mathrm{THz}$, centered at $0.36 \mathrm{THz}$. The successive stop-bands at higher frequencies are unresolvable due to the system bandwidth limit. The spectrum inside the stop-band of the $(H L)^{3} H$ filter is noisy, because the attenuation reaches the noise floor. The spectral transmittance for $(H L)^{4} H$ is not included, again due to the noise limit. The calculation is based on a characteristic matrix analysis.

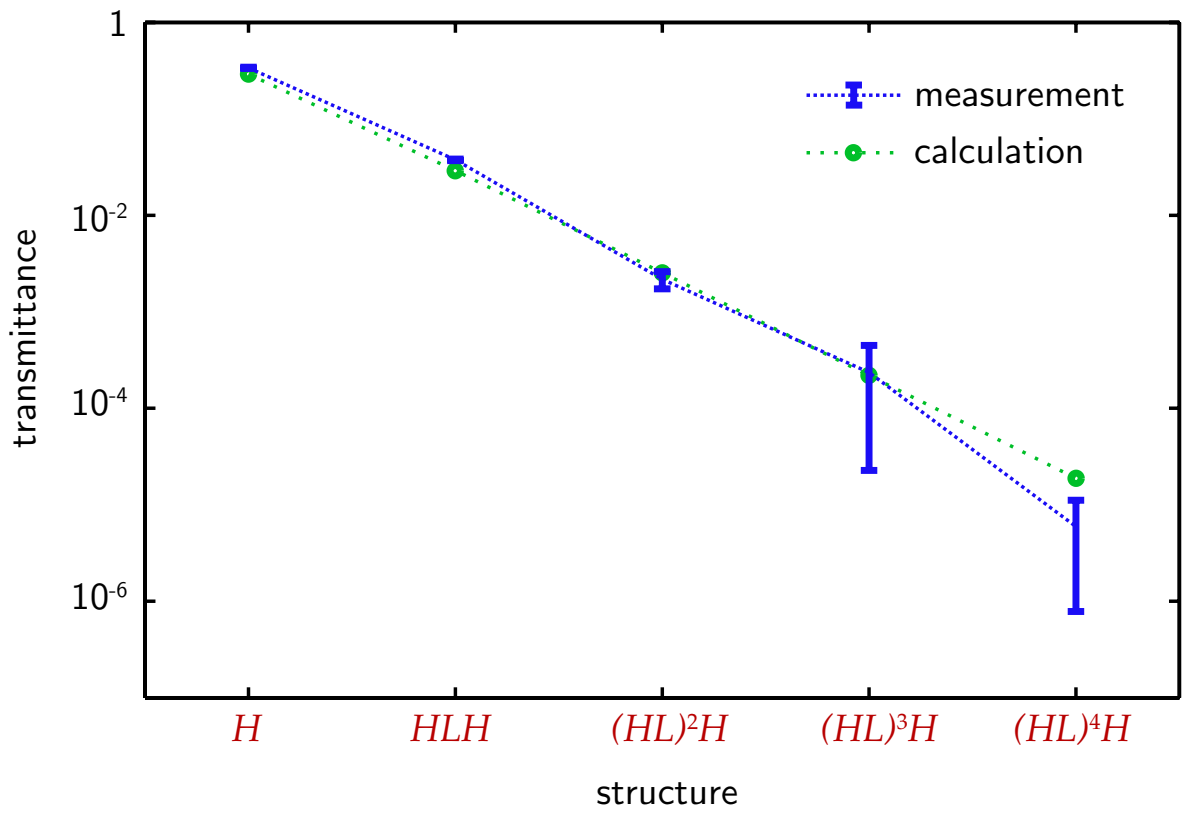

Figure 8. Transmittance of the interference filters at $0.36 \mathrm{THz}$. The attenuation at the middle of the first stop-band increases logarithmically with the number of periods. The transmittance starts to reach the noise level at $(H L)^{3} H$, where the measurement uncertainty is magnified. The calculation is based on Equation 13. The dotted lines merely assist interpretation. 


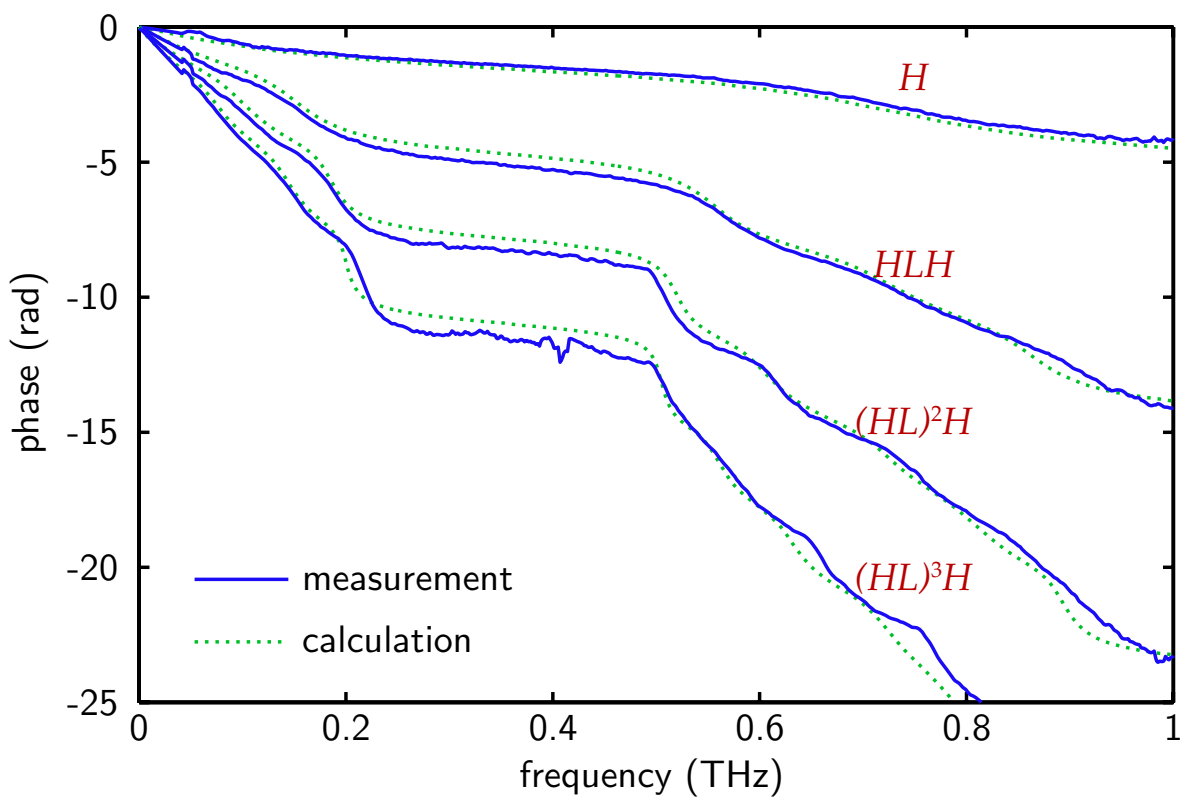

Figure 9. Phase response of the interference filters. The phases are anomalous inside the stop-band, i.e. between 0.2 and $0.5 \mathrm{THz}$. The spectral phase of $(H L)^{4} H$ is not included here due to the limit of the system dynamic range. The calculation is based on a characteristic matrix analysis.

following the number of periods. Due to the limited dynamic range of the system, it is not possible to render the second stop-band, which is beyond $0.9 \mathrm{THz}$.

The phase response is extracted using $\phi(\omega)=\angle E_{\mathrm{fil}}(\omega)-\angle E_{\mathrm{ref}}(\omega)-\omega L / c$. The $\omega L / c$ factor compensates the free space that occupies the structure's area with the thickness $L=a\left(l_{H}+l_{L}\right)+l_{H}$ in the reference measurement. The phase responses of the filters are shown in Figure 9. It is clear that the phase is anomalous in the stop-band, in the 0.2 to $0.5 \mathrm{THz}$ region.

In spite of the appearance of the first stop-band, the central frequency of the stop-band deviates from the expectation at $0.439 \mathrm{THz}$. This is more likely due to uneven surfaces of the air-gap rings, which result in thicker air layers. Hence, in the simulation, an adjustment to the material parameters is performed to compensate the structural thickness uncertainties. Through the fitting, using a characteristic matrix analysis, ${ }^{23}$ the thicknesses of the silicon wafers and air gaps are estimated at $60 \mu \mathrm{m}$ and $220 \mu \mathrm{m}$, respectively, whereas their indices of refraction remain unchanged. The simulation results accompany the measured transmittances and phase responses in Figures 7 and 9.

\section{CONCLUSION AND POTENTIAL EXTENSIONS}

A multilayer interference structure has an ability to manipulate the T-ray radiation in a frequency-selective manner. Despite the fact, most of the previous work focuses on its operation as a mirror, or more precisely, the structure is studied for its angle-dependent response to T-rays. A limited work presents its filtering characteristics following changes in structural parameters.

In this work, a set of the T-ray interference structures is designed to be operated as filter, in the transmission mode. Exploited in this work is a certain characteristic, namely the dependence of the stop-band attenuation on the number of filter layers. An explicit equation is derived, explaining the dependency in the logarithmic relation.

Realised according to the design, the filters are made from several ultrathin silicon wafers and air gaps, assembled in a retrofittable way. Characterised by transmission THz-TDS, the filters show an obvious stop-band spanning 0.2 to $0.5 \mathrm{THz}$ with its central frequency at $0.36 \mathrm{THz}$. The transmittance in the stop-band decreases at 


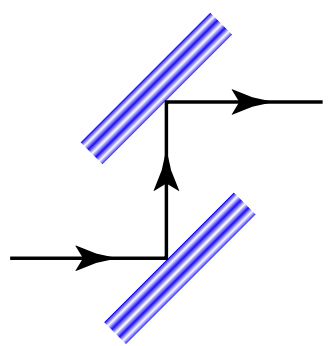

(a) two mirrors

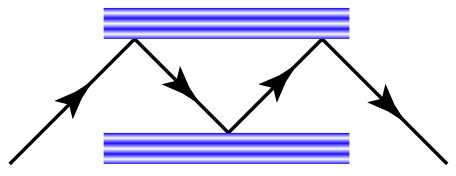

(b) three mirrors

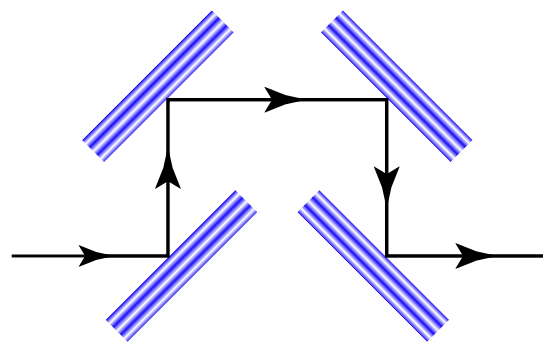

(c) four mirrors

Figure 10. Interference filter cascading in reflection arrangement. Possible schemes for cascading 2, 3 , and 4 interference filters (mirrors) in a reflection arrangement. In practice, the tilted angle of filters can be so small that the polarization dependence of filter response is negligible. After Shao and Dobrowolski. ${ }^{15}$

approximately an order of magnitude per silicon-air period, as predicted by the theory. A characteristic matrix analysis shows an agreement between the model and the measurements at the estimated thicknesses of the silicon and air gap of $60 \mu \mathrm{m}$ and $220 \mu \mathrm{m}$, respectively.

Although the filter pronounces an apparent stop-band, it is known that the filter response is spectrally periodic. This may not be practical if other responses such as if high-pass, low-pass or band-pass are required. As a potential extension, filter cascading schemes are introduced. They enable construction of an interference filter with an arbitrary response. By cascading a set of multilayer interference filters, it is possible to shape up any desired frequency response. Cascading of filters could be either in a transmission-mode arrangement, where the total transmittance, $\mathcal{T}_{\text {total }}$, is calculated from ${ }^{11}$

$$
\mathcal{T}_{\text {total }}=\mathcal{T}_{1} \mathcal{T}_{2} \ldots \mathcal{T}_{m}
$$

or a reflection-mode arrangement, ${ }^{15}$

$$
\mathcal{T}_{\text {total }}=\mathcal{R}_{1} \mathcal{R}_{2} \ldots \mathcal{R}_{m}
$$

where $\mathcal{T}_{m}$ and $\mathcal{R}_{m}$ are the individual transmittance and reflectance of each filter, respectively. Arrangement of a filter in a reflection mode is somewhat more difficult than arrangement in a transmission mode, as illustrated by some examples in Figure 10.

In the construction of the filter, although float-zone silicon is exceptionally transparent to T-rays, its cost is rather high. A number of other low-loss materials can substitute silicon in construction of the filter, with slightly inferior transparency. Bolivaret et al. ${ }^{27}$ suggests a range of low-loss yet low-cost dielectric materials, including steatite, alumina, titania loaded polystyrene, and zirconium-tin-titanate. The refractive indices of these materials span a wide range, which helps facilitate the design. Recently, in addition to those dielectrics, some polymers are introduced, e.g. picarin and cycloolefins. ${ }^{28}$ These polymers are transparent to both T-rays and visible light, and thus are attractive for some applications.

\section{Acknowledgement}

Thanks are due to Gretel Png and Brian Ng for their useful discussion and to Ian Linke for his excellent technical assistance on the polyethylene frame.

\section{REFERENCES}

1. W. Withayachumnankul, G. M. Png, X. X. Yin, S. Atakaramians, I. Jones, H. Lin, B. S. Y. Ung, J. Balakrishnan, B. W.-H. Ng, B. Ferguson, S. P. Mickan, B. M. Fischer, and D. Abbott, "Terahertz sensing and imaging," Proceedings of the IEEE 95(8), pp. 1528-1558, 2007. 
2. P. R. Griffths and J. de Haseth, Fourier Transform Infrared Spectroscopy, Wiley, New York, 1986.

3. J. S. Seeley, R. Hunneman, and A. Whatley, "Far infrared filters for the Galileo-Jupiter and other missions," Applied Optics 20(1), pp. 31-39, 1981.

4. A. F. Turner, L. Chang, and T. P. Martin, "Enhanced reflectance of reststrahlen reflection filters," Applied Optics 4(8), pp. 927-933, 1965.

5. K. R. Armstrong and F. J. Low, "Far-infrared filters utilizing small particle scattering and antireflection coatings," Applied Optics 13(2), pp. 425-430, 1974.

6. E. Özbay, E. Michel, G. Tuttle, R. Biswas, K. M. Ho, J. Bostak, and D. M. Bloom, "Double-etch geometry for millimeter-wave photonic band-gap crystals," Applied Physics Letters 65(13), pp. 1617-1619, 1994.

7. E. Özbay, E. Michel, G. Tuttle, R. Biswas, K. M. Ho, J. Bostak, and D. M. Bloom, "Terahertz spectroscopy of three-dimensional photonic band-gap crystals," Optics Letters 19(15), pp. 1155-1157, 1994.

8. K. F. Renk and L. Genzel, "Interference filters and Fabry-Perot interferometers for the far infrared," Applied Optics 1(5), pp. 643-648, 1962.

9. R. Ulrich, "Interference filters for the far infrared," Applied Optics 7(10), pp. 1987-1996, 1968.

10. C. Winnewisser, F. Lewen, and H.Helm, "Transmission characteristics of dichroic filters measured by THz time-domain spectroscopy," Applied Physics A: Materials Science ES Processing 66(6), pp. 593-598, 1998.

11. C. Winnewisser, F. Lewen, J. Weinzierl, and H. Helm, "Transmission features of frequency-selective components in the far infrared determined by terahertz time-domain spectroscopy," Applied Optics 38(18), pp. 3961-3967, 1999.

12. C. Winnewisser, F. T. Lewen, M. Schall, M. Walther, and H. Helm, "Characterization and application of dichroic filters in the 0.1-3 THz region," IEEE Transactions on Microwave Theory and Techniques 48(4), pp. $744-749,2000$.

13. D. Abbott and X.-C. Zhang, "Scanning the issue: T-ray imaging, sensing, and retection," Proceedings of the IEEE: A Special Issue on T-Ray Imaging, Sensing, and Retection 95(8), pp. 1509-1513, 2007.

14. P. R. Griffths, "Fourier transform infrared spectrometry," Science 222(4621), pp. 297-302, 1983.

15. J. Shao and J. A. Dobrowolski, "Multilayer interference filters for the far-infrared and submillimeter regions," Applied Optics 32(13), pp. 2361-2370, 1993.

16. R. Schiwon, G. Schwaab, E. Bründermann, and M. Havenith, "Far-infrared multilayer mirrors," Applied Physics Letters 83(20), pp. 4119-4121, 2003.

17. D. Turchinovich, A. Kammoun, P. Knobloch, T. Dobbertin, and M. Koch, "Flexible all-plastic mirrors for the THz range," Applied Physics A: Materials Science \& Processing 74(2), pp. 291-293, 2002.

18. P. Knobloch, D. Turchinovich, T. Dobbertin, and M. Koch, "Optical component." United States Patent: US 6,954,309, 2005.

19. N. Krumbholz, K. Gerlach, F. Rutz, M. Koch, R. Piesiewicz, T. Kürner, and D. Mittleman, "Omnidirectional terahertz mirrors: A key element for future terahertz communication systems," Applied Physics Letters 88, art. no. 202905, 2006.

20. F. Rutz, N. Krumbholz, L. Micele, G. de Portu, D. M. Mittleman, and M. Koch, "Improved dielectric mirrors for the THz frequency range," in Proc. SPIE Millimeter-Wave and Terahertz Photonics, D. Jäger and A. Stöhr, eds., 6194(1), (Strasbourg, France, April), art. no. 61940K, 2006.

21. F. Rutz, M. Koch, L. Micele, and G. de Portu, "Ceramic dielectric mirrors for the terahertz range," Applied Optics 45(31), pp. 8070-8073, 2006.

22. C. Jansen, F. Neubauer, J. Helbig, D. M. Mittleman, and M. Koch, "Flexible Bragg reflectors for the terahertz regime composed of polymeric compounds," in Conference Digest of the Joint 32th International Conference on Infrared and Millimeter Waves and 15th International Conference on Terahertz Electronics, pp. 984-986, (Cardiff, UK, September), 2007.

23. E. Hecht, Optics, Addison-Wesley, 2 ed., 1987.

24. S. J. Orfanidis, Electromagnetic Waves and Antennas, $2006 . \quad$ (Unpublished, available: http://www.ece.rutgers.edu/ orfanidi/ewa/).

25. D. Grischkowsky, S. Keiding, M. van Exter, and C. Fattinger, "Far-infrared time-domain spectroscopy with terahertz beams of dielectrics and semiconductors," Journal of the Optical Society of America B: Optical Physics 7(10), pp. 2006-2015, 1990. 
26. J. Dai, J. Zhang, W. Zhang, and D. Grischkowsky, "Terahertz time-domain spectroscopy characterization of the far-infrared absorption and index of refraction of high-resistivity, float-zone silicon," Journal of the Optical Society of America B: Optical Physics 21(7), pp. 1379-1386, 2004.

27. P. Bolivar, M. Brucherseifer, J. Rivas, R. Gonzalo, I. Ederra, A. Reynolds, M. Holker, and P. de Maagt, "Measurement of the dielectric constant and loss tangent of high dielectric-constant materials at terahertz frequencies," IEEE Transactions on Microwave Theory and Techniques 51(4 Part 1), pp. 1062-1066, 2003.

28. A. Sengupta, A. Bandyopadhyay, B. F. Bowden, J. A. Harrington, and J. F. Federici, "Characterisation of olefin copolymers using terahertz spectroscopy," Electronics Letters 42(25), pp. 1477-1479, 2006. 